University of Wollongong

Research Online

Faculty of Informatics - Papers (Archive)

Faculty of Engineering and Information

Sciences

17-10-2007

\title{
Peak-to-Average Power Ratio Performance of Interleaved Spread Spectrum OFDM Signals
}

\author{
P. Tu \\ University of Wollongong \\ Xiaojing Huang \\ University of Wollongong, huang@uow.edu.au \\ E. Dutkiewicz \\ University of Wollongong, eryk@uow.edu.au
}

Follow this and additional works at: https://ro.uow.edu.au/infopapers

Part of the Physical Sciences and Mathematics Commons

\section{Recommended Citation}

Tu, P.; Huang, Xiaojing; and Dutkiewicz, E.: Peak-to-Average Power Ratio Performance of Interleaved Spread Spectrum OFDM Signals 2007.

https://ro.uow.edu.au/infopapers/653

Research Online is the open access institutional repository for the University of Wollongong. For further information contact the UOW Library: research-pubs@uow.edu.au 


\title{
Peak-to-Average Power Ratio Performance of Interleaved Spread Spectrum OFDM Signals
}

\author{
Abstract \\ In this paper we propose an interleaved spread spectrum orthogonal frequency division multiplexing (ISS- \\ OFDM) transmission scheme and demonstrate its reduced peak-toaverage power ratio (PAR). The \\ proposed ISS-OFDM signal is realized by two steps. The first step is to modulate the data symbol by \\ complex exponential spreading and the second is to use an interleaving technique to spread the signal \\ spectrum as well as reduce the signal peak-to-average power ratio. The distinctive features of the \\ proposed method are that in the frequency domain the same data information is carried by a number of \\ subbands to effectively spread the signal spectrum and in the time domain the spread signal components \\ are not constructively added together so that signal peaks in the transmitted waveform are avoided. This \\ principle of PAR reduction is unique compared with other techniques such as selected mapping (SLM), \\ partial transmit sequences (PTS), and clipping. With the increase of the number of signal subbands, the \\ PAR performance is improved significantly.

\section{Disciplines} \\ Physical Sciences and Mathematics

\section{Publication Details} \\ This conference paper was originally published as P. Tu, Huang, X, Dutkiewicz, E, Peak-to-Average Power \\ Ratio Performance of Interleaved Spread Spectrum OFDM Signals, International Symposium on \\ Communications and Information Technologies ISCIT 2007, 17-19 Oct, 82-86.
}




\title{
Peak-to-Average Power Ratio Performance of Interleaved Spread Spectrum OFDM Signals
}

\author{
Pingzhou Tu, Xiaojing Huang, Eryk Dutkiewicz \\ Email: \{pt015, huang, eryk\}@uow.edu.au \\ School of Electrical, Computer and Telecommunications Engineering \\ University of Wollongong, Australia
}

\begin{abstract}
In this paper we propose an interleaved spread spectrum orthogonal frequency division multiplexing (ISS-OFDM) transmission scheme and demonstrate its reduced peak-toaverage power ratio (PAR). The proposed ISS-OFDM signal is realized by two steps. The first step is to modulate the data symbol by complex exponential spreading and the second is to use an interleaving technique to spread the signal spectrum as well as reduce the signal peak-to-average power ratio. The distinctive features of the proposed method are that in the frequency domain the same data information is carried by a number of subbands to effectively spread the signal spectrum and in the time domain the spread signal components are not constructively added together so that signal peaks in the transmitted waveform are avoided. This principle of PAR reduction is unique compared with other techniques such as selected mapping (SLM), partial transmit sequences (PTS), and clipping. With the increase of the number of signal subbands, the PAR performance is improved significantly.
\end{abstract}

\section{INTRODUCTION}

Orthogonal frequency division multiplexing (OFDM) technique has attracted significant attention due to its simple implementation by employing the Inverse Fast Fourier Transform (IFFT) operation and its extended symbol duration to combat intersymbol interference (ISI). It has found wide applications such as digital audio/video broadcasting (DAB/DVB), wireless local area networks (WLAN) (e.g., IEEE802.11a/g and Hiperlan II), and wireless personal area networks (WPAN) (e.g., MB-OFDM) [1,2]. More recently, OFDM has been considered as one of the most promising techniques to be applied to the adaptive frequency sharing management systems in prospective cognitive radio communications [3].

However, OFDM has some drawbacks which prevent it from being used for low power and low cost applications. One of the major disadvantages is the large peak-to-average power ratio (PAR) of the transmitted signal, which renders a straightforward implementation very costly and inefficient. Especially, when the number of subcarriers becomes large, the PAR of the transmitted signal is sometimes unacceptable. The cause of a large PAR in conventional OFDM system is partially related to how the OFDM signal is formed. Consider the case when the signal frequency band is divided into $N$ subcarriers and the frequency separation between subcarriers is $1 / T$, where $T$ is the OFDM symbol time duration. The data to be transmitted are split into streams, each of which modulates a corresponding subcarrier. The $N$ streams are modulated in parallel on closely-spaced subcarriers. The practical implementation of this process is performed using IFFT to generate a sampled version of the composite time signal. When the large number of signals from different subcarriers is added together, the signals with the same phases at the same time instants will produce high amplitude peaks. Compared with the average signal power, the instantaneous power of these peaks is very high, making the amplifier of the transmitter work in non-linear range, which significantly degrades the system performance [4].

In order to reduce the PAR and improve OFDM system performance, some different algorithms have been proposed, such as selected mapping (SLM), partial transmit sequences (PTS) [5,6], interleaved OFDM (IOFDM) [7], and block coding and clipping with filtering [8]. For the SLM method, the basic idea is to have $N$ statistically independent vectors representing the same information before modulation. After modulation and filtering, the time domain symbol with the lowest PAR is selected for transmission. PTS is based on the same principle as that of SLM, except that the transformation vectors have a different structure. The clipping technique is the most straightforward way to deal with the PAR problems, by just clipping the signal with amplitudes over a certain threshold, but it results in a large amount of noise. These methods, except for clipping, are the same in principle, i.e., they generate some redundant signals bearing the same information and then select the best vector with the lowest PAR. A common problem for these algorithms is that they need to transmit a number of side information so that the receiver can recover the original information, which causes the transmission data rate loss. Another main drawback is the huge cost of computational complexity for choosing a good vector with the lowest PAR [9].

In this paper, we propose an interleaved spread spectrum orthogonal frequency division multiplexing (ISS-OFDM) signal by spread spectrum modulation and interleaving techniques with significant PAR reduction. The ISS-OFDM signal is generated by two steps. The first step is to modulate the data information by complex exponential spreading and the second is to spread the signal spectrum by interleaving [10]. At the transmitter, each data symbol modulates the corresponding subcarriers multiple times, and several different samples bearing the same data information can be obtained. The replicas of the same data information are interleaved into a serial sequence and then transmitted in different time slots. Assume 


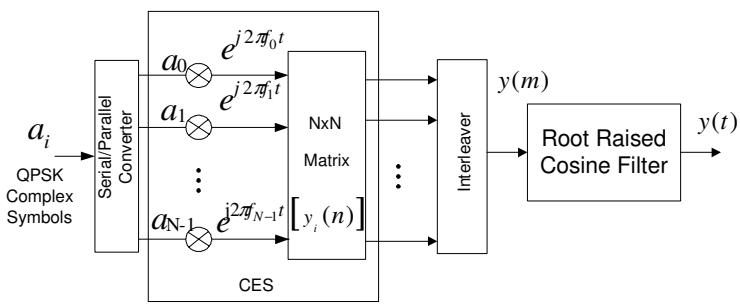

Fig. 1. Transmitter model.

that $N$ data symbols in parallel modulate $N$ subcarriers to produce $N$ samples at each time slot. The produced $N$ samples are not added together. Instead, they are interleaved pseudo randomly according to a certain pseudorandom code. Then, they are transmitted on different time slots respectively. After this spread spectrum modulation, an $N \times N$ matrix of data samples is generated, and then interleaved [7,10,11]. In this way, the number of the total samples in one ISS-OFDM symbol increases greatly, which results in reduced signal power spectrum density if the total signal energy remains unchanged. At the same time, the PAR is reduced significantly. At the receiver, the received signal replicas in different subbands can be used to recover the data information by employing Fast Fourier Transform (FFT) without using any side information.

In the rest of this paper, we first describe the transmitter model of the ISS-OFDM system in Section II. Then detailed ISS-OFDM signal generation and PAR reduction principle are given in Section III. In Sections IV we evaluate the PAR performance and present the simulation results. Finally, concluding remarks are drawn in Section V.

\section{SYSTEM MODEL}

Fig. 1 displays the transmitter model for the ISS-OFDM system, which consists of the QPSK mapper, complex exponential spreading (CES) module, interleaver and filter. The function of the transmitter is to generate a transmitted signal, called ISS-OFDM signal. In the transmitter, the complex QPSK data symbol sequence is first divided into an $N$ by 1 vector, where $N$ is the number of subcarriers. Then, the vector with $N$ data symbols modulates $N$ subcarries in parallel by using CES operation, by which each data symbol modulates its corresponding subcarrier. After the modulation, an $N \times N$ matrix is formed, the modulated subcarriers in the matrix are interleaved and placed on different time slots to form a serial sequence. The sequence is pulse shaped by passing through a root raised cosine filter and thus an ISS-OFDM signal $y(t)$ is generated.

Time domain samples of the transmitted signal in the equivalent complex valued low-pass domain are approximately Gaussian distributed due to the statistical independence of carriers. Resulting PAR of $y(t)$ can be written as

$$
\xi=\frac{\max |y(t)|^{2}}{E\left[|y(t)|^{2}\right]}
$$

where $\max |y(t)|^{2}$ is the maximum instantaneous power of the ISS-OFDM signal and $E\left[|y(t)|^{2}\right]$ is the expected value of $|y(t)|^{2}$.

Note that the actual PAR of the continuous-time ISS-OFDM signal can not be determined by using Nyquist sampling rate. Otherwise, the signal peaks are often missed and PAR reduction estimates are unduly optimistic. In order to evaluate the PAR performance of the transmitted signal, we oversample the signal by a factor of four, which is sufficient to produce accurate PAR [11].

\section{TRANSMITTED SIGNAL GENERATION AND PAR REDUCTION}

\section{A. Complex Exponential Spreading}

For convenience of description, we will discuss signal generation of one ISS-OFDM symbol only. Let $a_{i}(i=$ $0,1, \cdots, N)$ denote the $i^{t h}$ symbol of the $N$ QPSK complexvalued symbols required to be transmitted. The $N$ QPSK symbols are modulated by CES modulator, and then interleaved. Assume that the ISS-OFDM symbol period is $T_{s}$, $f_{i}=\frac{i}{T_{s}}$ denotes the $i^{t h}$ subcarrier frequency of the $N$ orthogonal subcarriers, and $a_{i}$ modulates the $i^{\text {th }}$ subcarrier at time $t=\frac{n}{N} T_{s}, n=0,1, \cdots, N-1$, where $n$ is the time index. The modulated symbol on the $i^{\text {th }}$ subcarrier and at the $n^{\text {th }}$ time instant is written as follows,

$$
\begin{aligned}
y_{i}(n) & =a_{i} e^{j 2 \pi f_{i} t} \\
& =a_{i} e^{j 2 \pi n i / N} .
\end{aligned}
$$

In an ISS-OFDM symbol period $T_{s}$, each element of the $N \times 1$ data symbol vector modulates the $N$ samples of the same corresponding subcarrier $N$ times. $N$ elements in the vector generate an $N \times N$ sample matrix after CES modulation. Mathematically, the matrix can be expressed as

$$
\begin{gathered}
{\left[y_{i}(n)\right]=\left(\begin{array}{c}
y_{0}(0), y_{0}(1), \cdots, y_{0}(N-1) \\
y_{1}(0), y_{1}(1), \cdots, y_{1}(N-1) \\
\cdots \cdots \cdots \\
y_{N-1}(0), y_{N-1}(1), \cdots, y_{N-1}(N-1)
\end{array}\right)=} \\
\left(\begin{array}{c}
a_{0} e^{j 2 \pi 0 * 0 / N}, a_{0} e^{j 2 \pi 0 * 1 / N}, \cdots, a_{0} e^{j 2 \pi 0 *(N-1) / N} \\
a_{1} e^{j 2 \pi 1 * 0 / N}, a_{1} e^{j 2 \pi 1 * 1 / N}, \cdots, a_{1} e^{j 2 \pi 1 *(N-1) / N} \\
\cdots \cdots \cdots \\
a_{N-1} e^{j 2 \pi 0 * 0 / N}, a_{N-1} e^{j 2 \pi 0 * 1 / N}, \cdots, a_{N-1} e^{j 2 \pi 0 *(N-1) / N}
\end{array}\right) .
\end{gathered}
$$

Let $W_{N}=e^{-j \frac{2 \pi}{N}}$, and $\left[W_{N}^{-n i}\right]=\left[e^{j \frac{2 \pi}{N} n i}\right]$, where $W_{N}^{-n i}$ denote the $i^{\text {th }}$ sub-carrier at the $\frac{n}{N} T_{s}$ time instant, and

$$
\operatorname{Diag}\left(a_{i}\right)=\left(\begin{array}{c}
a_{0}, 0, \cdots, 0 \\
0, a_{1}, 0, \cdots, 0 \\
\cdots \\
0,0, \cdots, a_{N-1}
\end{array}\right) .
$$

Thus, Equation (3) can be rewritten as, 


$$
\begin{gathered}
{\left[y_{i}(n)\right]=} \\
\left(\begin{array}{c}
a_{0} W_{N}^{-0 * 0}, a_{0} W_{N}^{-0 * 1}, \ldots, a_{0} W_{N}^{-0 *(N-1)} \\
a_{1} W_{N}^{-1 * 0}, a_{1} W_{N}^{-1 * 1}, \ldots, a_{1} W_{N}^{-1 *(N-1)} \\
\ldots \ldots \\
a_{N-1} W_{N}^{0}, a_{N-1} W_{N}^{-(N-1) * 1}, \ldots, a_{N-1} W_{N}^{-(N-1) *(N-1)}
\end{array}\right) \\
=\operatorname{Diag}\left(a_{i}\right) \times\left[W_{N}^{-n i}\right] .
\end{gathered}
$$

Equation (4) indicates one way of implementing CES modulation, by which the $N$ transmitted data symbols at the input of the modulator are rearranged into the diagonal elements of a diagonal matrix. Since the input signal of the modulator is in the form of a diagonal matrix, the output signal is an $N \times N$ sample matrix as denoted in Equation (3).

\section{B. Phase Shifting and Interleaving}

In order to spread the signal spectrum and at the same time reduce the probability of occurrence of large signal peaks in the transmitted signal, the samples in the $N \times N$ matrix are interleaved to form a serial sequence of length $N^{2}$ in the time domain. Each of the samples is placed on a certain time slot. Here, different interleaving algorithms can be employed such as pseudo random interleaving, periodic interleaving and convolutional interleaving. In the following, we discuss the periodic interleaving only. The periodic interleaving can be realized by taking out the $N$ samples in columns of the matrix and then placing the $N$ modulated samples on different time slots respectively. In fact, the effect of the interleaving also introduces certain phase shifts in different subcarriers. For instance, the $i^{t h}$ subcarrier of the $N$ subcarriers is shifted by $i \cdot \tau$ in time, where $\tau=\frac{T_{s}}{N^{2}}$ is the sampling interval. That is, the phase of the $i^{t h}$ subcarrier is shifted $\frac{2 \pi}{N^{2}} i$. After interleaving, the $m^{\text {th }}$ sample in the ISS-OFDM symbol, denoted by $y(m)$, $m=n N+i=0,1, \cdots, N^{2}-1$, can be mathematically expressed as follows

$$
y(m)=\sum_{i=1}^{N} \sum_{n}^{N} y_{i}(n) \delta[m-i-n N]
$$

where $\delta[m-n N-i]=\left\{\begin{array}{l}1, \text { for } m=n N+i \\ 0, \text { for } m \neq n N+i\end{array}\right.$ is the unit impulse. Referring to Equation (2) and assuming $m=n N+i$, $y(m)$ can be simplified as the form

$$
\begin{aligned}
& y(m)=y(n N+i) \\
& =y_{i}(n) \\
& =a_{i} e^{j 2 \pi n i / N}
\end{aligned}
$$

where $m=n N+i$, and $n, i=0,1, \cdots, N-1$.

From the above we see that instead of being superimposed together the $N$ modulated samples are interleaved periodically and then added together in different time instants. Thus, one ISS-OFDM symbol with $N^{2}$ samples is produced in the symbol duration $T_{s}$, and the number of samples is increased by $N$ times. The sampling rate is increased to $N^{2} / T_{s}$. Consequently, the signal spectrum is spread $N$ times and

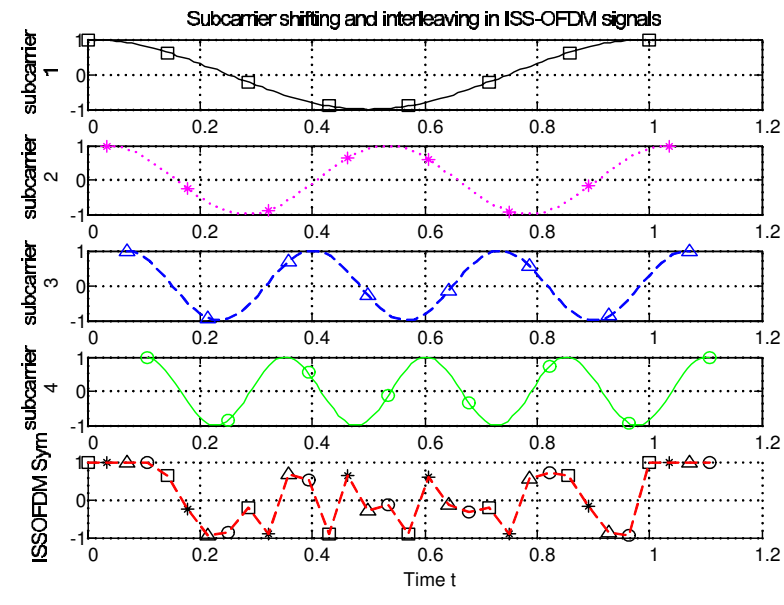

Fig. 2. Subcarrier shifting and interleaving in ISS-OFDM signals with subcarrier number $\mathrm{N}=4$.

the power spectrum density decreases to $1 / N$ for the same transmitted signal energy.

Fig. 2 displays the subcarrier shift in the time domain for the ISS-OFDM transmitted signal when the number of subcarriers $N$ is equal to 4 . It can be seen clearly that the modulated samples on the $i^{t h}(i=1,2,3,4)$ subcarrier are shifted $i$ intervals, so that the number of samples increases to $N^{2}$ from the original number $N$ after interleaving. We can observe that the maximum power of the ISS-OFDM transmitted signal is 1 , and there is no increase in the maximum power compared to the individual subcarrier.

Fig. 3 shows the four subcarriers and their spectrum expansion in frequency domain. The subcarrier spectrum expansion is due to the subcarrier shifting and interleaving in the time domain. It is expected that after filtering the probability of the peak signal power above a certain threshold will be decreased greatly compared with the conventional OFDM system. The higher the number of subcarrriers is, the wider the subcarrier spectrum expands.

\section{Comparison of Transmitted Signals between ISS-OFDM and Conventional OFDM}

If we compare the transmitted signal power of the ISSOFDM with that of conventional OFDM systems, the difference is obvious. Assume that the total power of the transmitted signal is $P$ and $N$ subcarriers are modulated by $N$ QPSK symbols. In conventional OFDM systems, there are $N$ modulated samples produced within one OFDM symbol duration, and the power on each sample is $\frac{P}{N}$. In the ISS-OFDM system, due to the signal spectrum spreading, there are $N \times N$ samples produced within the same OFDM symbol duration, and the power on each sample is $\frac{P}{N \times N}$. It is observed that the power on each individual sample in ISS-OFDM transmitted signal is reduced to $\frac{1}{N}$ of the conventional OFDM signal.

The difference in the signal power spectrum density between the ISS-OFDM signals and OFDM signals can be also seen 


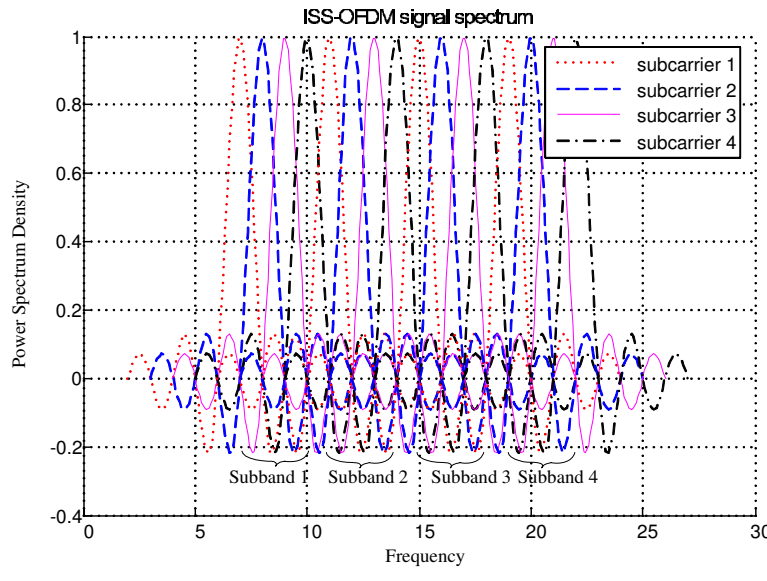

Fig. 3. ISS-OFDM signal spectrum with subcarrier number $\mathrm{N}=4$.

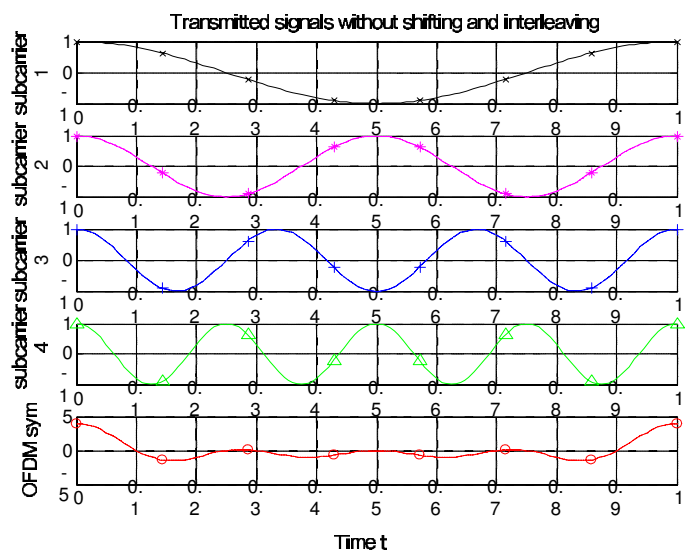

Fig. 4. Transmitted signal without shifting and interleaving in conventional OFDM system with subcarrier number $\mathrm{N}=4$.

very clearly in Fig. 4, which shows the signal modulation process and the resulting transmitted signals in the conventional OFDM system. We see that the at some time instants the subcarriers are combined constructively and that the maximum power of transmitted signal is 4 , which is 4 times larger than that of one individual subcarrier. It is expected that the probability of occurrence of large signal peaks increases greatly after pulse shaping by the transmitter filter.

\section{SIMULATION RESULTS AND ANALYSIS}

\section{A. ISS-OFDM Signals under Different Spreading Factors}

Assume that the data symbol mapping scheme uses QPSK and the number of subcarriers $N=32$. The generated ISSOFDM signal has $N^{2}$ samples and contains $N$ subbands, each of which contains $N$ subcarriers and carries the same data information. The transmitter is designed so that $M$ subbands are selected, $M(M=0,1, \cdots, N)$ respectively, and thus the transmitted signal contains $M$ subbands. This means that the
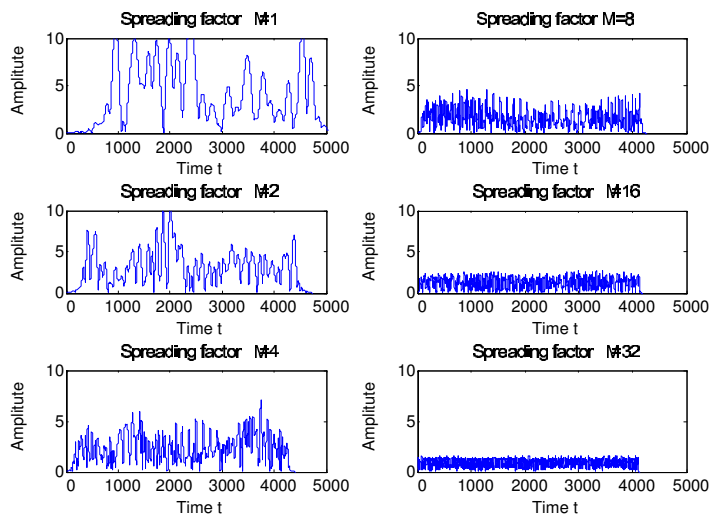

Fig. 5. Transmitted signal waveforms with different spreading factors.

spectrum spreading factor is $M$. Fig. 5 shows the transmitted ISS-OFDM signal waveforms with various spectrum spreading factors $M=1,2,4,8,16$ and 32 respectively. We assume that data symbol has the same energy for different spreading factors. It can be observed from Fig. 5 that with the increase of the spreading factor the signal amplitude (power) necessary for achieving the same bit error performance decreases.

\section{B. PAR Simulation Results and Analysis}

Under the same assumptions as above, we select the signal bandwidth to contain $M=1,2,4,8,16$ and 32 subbands respectively ( $M$ is the spreading factor), and the filtered signal is oversampled by a factor of four, which is commonly used to estimate the PAR of an analog signal from its samples. When $M=1$, there is only one signal subband to be allowed to pass through the filter, resulting in a conventional OFDM signal. When $M=2,4,8,16$ and 32 , respectively, i.e., the spreading factor is $M=2,4,8,16$ and 32 , the signal contains $M=2,4,8,16$ and 32 subbands respectively.

According to (1) we can compute the PAR performance curves. Fig. 6 shows the PAR performance of the ISS-OFDM transmitted signal when PAR exceeds a certain threshold PAR0 with the increase of the spectrum spreading factor $M$ from 1 to 32 .

It can be seen from Fig. 6 that with the increase of the number of subbands passing through the filter, the PAR performance is improved considerably. The most right-handside curve shows the PAR performance of ISS-OFDM signal when $M$ is equal to 1 , which is the same as that of conventional OFDM signal. As $M$ increases to 2, 4, 8, 16, and 32 respectively, the PAR gains are $0.5 \mathrm{~dB}, 1.5 \mathrm{~dB}, 3.5 \mathrm{~dB}, 5 \mathrm{~dB}$ and $7 \mathrm{~dB}$ respectively.

\section{Computational Complexity}

Computational complexity for generating one ISS-OFDM symbol can be also obtained. If we ignore the complexity of the complex-valued addition, the total number of complex multiplications for one ISS-OFDM symbol is $\frac{1}{2} N \times N \log _{2}^{N}$. 


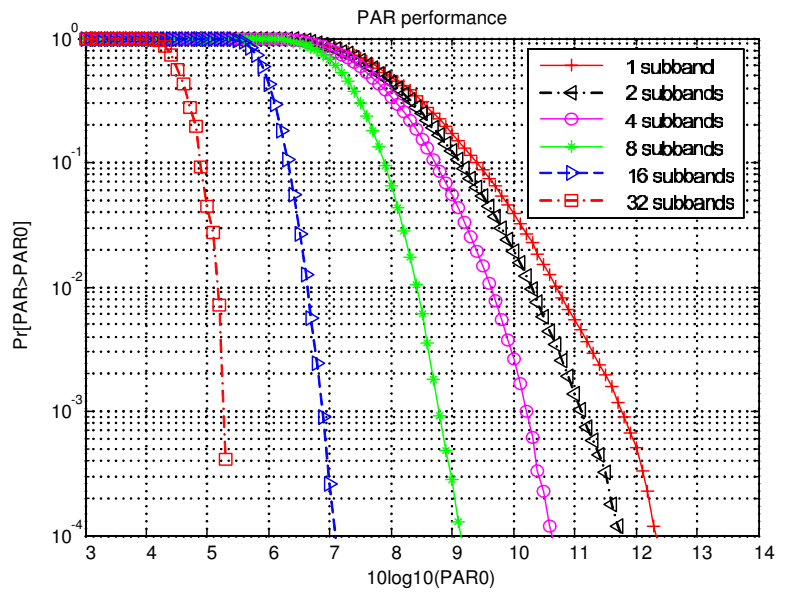

Fig. 6. PAR performance for ISS-OFDM signals with different numbers of subbands.

For a conventional OFDM, one IFFT operation is employed so that the total number of complex multiplications is $\frac{1}{2} N \log _{2}^{N}$. The computational complexity for generating an ISS-OFDM symbol is increased $N$ times. That is, significant PAR gain is obtained in ISS-OFDM signals at the cost of a slight increase of computational complexity.

\section{CONCLUSION}

In this paper, we have investigated the PAR performance of the ISS-OFDM system. It has been showed that the combination of complex exponential spreading and interleaving not only spreads the signal spectrum but also reduces the PAR of the resulting ISS-OFDM signal significantly. It has been also shown that the PAR performance can be improved up to $2 \mathrm{~dB}$ once an additional subband is added to the signal spectrum. Compared with other previous PAR reduction algorithms, ISSOFDM also effectively realizes a spread spectrum so that it does not degrade the range of communications or need to transmit side information. The proposed ISS-OFDM system can be used for ultra-wideband communications. It is also expected to be used in cognitive radio adaptive modulation techniques.

\section{REFERENCES}

[1] O. Edfors, M. Sandell, J. J. V. D. Beek, S.K.Wilson and P. O. Borjesson, "OFDM Channel Estimation by Singular Value Decomposition," IEEE Transactions on Communications, vol. 46, no. 7, pp. 931-938, 1998.

[2] R. Novak and W. A. Krzymien, "Diversity Combining Options for Spread Spectrum OFDM in Frequency Selective Channels," Wireless Communications and Networking Conference, vol. 1, pp. 308-314, 2005.

[3] S. Haykin, "Cognitive Radio:Brain-Empowered Wireless Communications," IEEE Journal on Selected Areas in Communications, vol. 23, no. 2, pp. 201-219, 2005.

[4] J. G. Proakis, Digital Communications, 4 ed. New York, USA: Mc Graw Hill, 2000.

[5] A. D. S Jayalath and C.Tellambura, "Use of data permutation to reduce the peak-to-average power ratio of an OFDM signal," Wireless Communications and Mobile Computing, vol. 2, pp. 187-203, 2002.
[6] C. Schurgers and M. B. Srivastava, "A Systematic Approach to Peakto-Average Power Ratio in OFDM," Proc. SPIE, vol. 4474, no. 11, pp. 454-464, 2001.

[7] V. G. S. Prasad and K. V. S. Hari, "Interleaved Orthogonal Frequency Division Multiplexing System," Acoustics, Speech, and Signal Processing, vol. 3, p. III-2745-III-2748, 2002.

[8] S. B. Slimane, "Peak-to-Average Power Ratio Reduction of OFDM Signals Using Broadband Pulse Shaping," http://citeseer. ist. psu. edu/438615. html, pp. 889-893, 2002.

[9] L. Wan and V. K. Dubey, "BER Performance of OFDM system Over Frequency Nonselective Fast Ricean Fading Channels," IEEE Transaction Letters, vol. 5, no. 1, pp. 19-21, 2001.

[10] P. Tu, X. Huang and E. Dutkiewicz, "A Novel Approach of Spread Spectrum in OFDM Systems," International Symposium on Communications and Information Technologies, 2006, pp. 487-491, 2006.

[11] A. D. S Jayalath and C. Tellambura, "Interleaved PC-OFDM to Reduce the Peak-to-average Power Ratio," Advanced Signal Processing for Communication Systems, Springer Netherlands, 2007, pp. 239-250. 